\title{
ESTIMACIÓN DE LA TEMPERATURA BASAL DEL "GLACIAR NORTE" DEL VOLCÁN CITLALTÉPETL, MÉXICO. MODELO PARA DETERMINAR LA PRESENCIA DE PERMAFROST SUBGLACIAR
}

\author{
Víctor Hugo Soto Molina \\ Universidad Nacional Autónoma de México \\ islaveracruz@gmail.com \\ ORCID iD: http://orcid.org/0000-0003-1633-0752 \\ Hugo Delgado Granados \\ Universidad Nacional Autónoma de México \\ hdg@geofísica.unam.mx \\ ORCID iD: http://orcid.org/0000-0001-5263-7968 \\ Guillermo Ontiveros González \\ Universidad Nacional Autónoma de México \\ gontiverosg@gmail.com \\ ORCID iD: http://orcid.org/0000-0001-8286-6737
}

Recibido: 01/09/2018; Aceptado: 25/06/2019.

\begin{abstract}
Cómo citar este artículo/Citation: Soto Molina, V. H., Delgado Granados, H. y Ontiveros González, G. (2019). Estimación de la temperatura basal del "Glaciar Norte" del volcán Citlaltépetl, México. Modelo para determinar la presencia de permafrost subglaciar. Estudios Geográficos, 80 (287), e019. https://doi.org/10.3989/estgeogr.201936.016
\end{abstract}

RESUMEN: Se estudian las condiciones de temperatura y la conductividad del hielo del "Glaciar Norte" del volcán Citlaltépetl con el objeto de determinar la existencia de permafrost basal. A partir de registros de temperatura cercanos a la superficie se estimó la temperatura media en estado estacionario del interior del "Glaciar Norte" mediante un método sinusoidal que utiliza las propiedades termo-conductoras del hielo. A través de su perfil de temperatura se obtuvo una aproximación a las condiciones térmicas del principal glaciar de México. Los resultados podrían sugerir la existencia de un permafrost basal, el cual sería producto del aislamiento del hielo y de la conducción térmica por contacto directo entre el hielo y el substrato.

PALABRAS CLAVE: Glaciar; permafrost basal; conducción de calor; temperatura; estado estacionario.

\section{ESTIMATION OF THE TEMPERATURE IN THE BOTTOM OF THE “GLACIAR NORTE” OF THE CITLALTE- PETL VOLCANO, MEXICO. MODEL TO DETERMINE THE PRESENCE OF SUBGLACIAL PERMAFROST}

ABSTRACT: The conditions of temperature and the "Glaciar Norte" ice conductivity in the Citlaltepetl volcano are studied with the aim to determinate its basal permafrost. From temperature records close to surface, the temperature average in internal steady-state of "Glaciar Norte" was estimated using a sinusoidal method, which manage the thermal-conductive properties of ice. Through its temperature profile, an approach to the thermal conditions of Mexico's main glacier was obtained. The results may suggest the existence of a basal permafrost, which would be due to the ice insulation and the thermal conduction by direct contact between ice and substratum.

KEY WORDS: Glacier; basal permafrost; thermal conduction; temperature; steady-state. 


\section{INTRODUCCIÓN}

Dos de los elementos de la criósfera que pueden existir en altas montañas, además de la nieve estacional, son el permafrost y los glaciares. El permafrost se define como un suelo, formación superficial o substrato afectado por la dinámica de hielo-deshielo hasta una profundidad de $-60 \mathrm{~cm}$ (French, 1976, p. 47). Desde el punto de vista del régimen térmico, estas condiciones implican una "temperatura media anual por debajo de $0^{\circ} \mathrm{C}$ " (French, 2007, p. 83) o de $-2^{\circ} \mathrm{C}$ (French y Shur, 2010; Barry, 2017), durante al menos dos años continuos. Al existir una estrecha correlación aire-suelo, desde el punto de vista térmico (Arya, 2001), es común la presencia de permafrost en ambientes con una temperatura media anual del aire inferior a -1 o $-2^{\circ} \mathrm{C}$ (Demek, 1994). Por otra parte, un glaciar de montaña es una masa dinámica de hielo formada a partir de la acumulación y compactación de los cristales de nieve a través de los años; sus propiedades físicas varían durante el tiempo debido a procesos internos como el flujo vertical y horizontal de su masa, la compactación y densidad, por la generación de calor por fricción y por la presión de sí mismo (Ceballos-Liévano et al., 2012).

La conjugación de los procesos mecánicos de un glaciar provoca, entre otras respuestas, que la disponibilidad de la energía solar neta en su superficie sea distribuida de manera distinta en todo momento, ocasionando que cada glaciar sea único y al mismo tiempo varíe entre uno y otro estado prácticamente de un día para otro. Por tal razón, pretender calcular la distribución de la temperatura en su interior, así como otras variables físicas resulta una tarea compleja (Blatter y Haeberli, 1984) y su valor estimado sólo sería válido para un momento dado. No obstante lo anterior, al igual que como sucede con distintos procesos geofísicos, si se cuenta con datos observados de un periodo considerablemente largo es posible estimar valores promediados con la finalidad de contar con una dinámica y condiciones internas medias.

Una de las interacciones de los glaciares con su entorno radica en la que tiene que ver con la protección aislante del hielo entre la atmósfera y el lecho sobre el cual descansa, la cual es debida principalmente a la capacidad de albedo del hielo que oscila entre 0.35 en glaciares inestables y relativamente sucios (Grenfell, 2011) y 0.55 en hielo limpio (Van den Broeke et al., 2011), en comparación con el 0.25 del suelo desnudo (Ahrens, 2006) y 0.22 en roca sólida (Ramírez y Muñoz, 2012); en segunda instancia tiene que ver su capacidad conductora de calor. El albedo repercute de manera directa en la cantidad de energía radiativa neta $R_{N}$ en la superficie que a su vez se traduce en un flujo limitado de calor hacia el interior $H_{G}$ del cuerpo de hielo y al mismo tiempo de la base sobre la que se posiciona.

La ecuación del balance de energía $B E$ gobierna la distribución de la $R_{N}$ en la superficie y el almacenamiento o liberación de calor por debajo de ésta (Rivas y Ocampo, 2009) en su forma más simple, despreciando la energía de la precipitación por su escasa aportación (Rivas y Caselles, 2004), está dada por la expresión:

$$
B E=R_{N}+H_{G}+H_{L}+H_{S}=0
$$

Donde la suma de los flujos turbulentos latente $H_{L}$ y sensible $H_{S}$ más la radiación neta incidente $R_{N}$ junto con el flujo interno $H_{G}$ habrán de dar siempre 0 .

Una vez que parte de la $R_{N}$ disponible en la superficie glaciar se ha ocupado en la evaporación y sublimación mediante el componente $H_{L}$ y el calentamiento del aire por medio de $H_{S^{\prime}}$ la diferencia de $H_{G}$ habrá de buscar el equilibrio térmico en la columna de hielo. En condiciones generales la distribución de la temperatura interna depende del gradiente térmico que está también equilibrado por el flujo geotérmico, el calor friccional y el calentamiento por presión. No obstante, el calor geotérmico solo comienza a ser perceptible por debajo de $\sim 15$ de metros de profundidad (Andújar-Márquez et al., 2016) una vez que deja de influir la energía exterior; por lo que en este caso, más que introducirse como flujo de calor hacia el interior del glaciar, su función es la del amortiguamiento a cierta profundidad del lecho rocoso entre el frío superior y el calor inferior; de esta manera la temperatura en su base sería función del balance calórico entre la capa superior de hielo y la sub-superficie del lecho (Bennett y Glasser, 2011). De este modo, la temperatura basal del glaciar es transferida por conducción hacia el lecho sobre el que descansa, hasta lograr un equilibrio térmico entre los dos elementos. Esta transferencia depende directamente de la capacidad calórica, la conductividad térmica y la densidad, cuya relación determina la difusividad termal o velocidad en la que se transmite el calor dentro del cuerpo de hielo.

La complejidad de las características de un glaciar de montaña es muy distinta a los cuerpos de hielo polares debido a la altitud, relieve, inclinación, flujo y balance de masa diferenciales a lo largo del tiempo; esto obliga a estimar sus propiedades físicas basadas en un estado estacionario (Blatter y Haeberli, 1984), entre ellas la distribución de la temperatura interna. Esta 
distribución está dada en sentido horizontal y vertical, no obstante, el gradiente advectivo suele ser tan pequeño que es mayormente desestimado en todos los casos. El modelo de cálculo de la temperatura de un glaciar en estado estacionario representa la forma más sencilla de estimación y brinda una buena aproximación del comportamiento térmico en su interior (Cuffey y Paterson, 2010). Considerando lo anterior, el flujo de energía al interior del glaciar y por consecuencia de su temperatura estaría regido por la ecuación de Fourier en su modo más simple como:

$$
Q=k A \frac{T_{i-T_{f}}}{Z}
$$

Donde $Q$ es el flujo de energía en Watts $/ \mathrm{m}^{2}, k$ es la conductividad térmica del hielo por área $A ; T_{i}$ y $T_{f}$ son las temperaturas inicial y final respectivamente y $Z$ representa la profundidad. Sin embargo, al pretender realizar el cálculo del perfil de temperaturas, este método lo considera de manera lineal desde la superficie hasta la base sin considerar la fuerte incidencia que tiene la radiación y las condiciones de temperatura del aire sobre los primeros metros por debajo de la superficie y que ocasiona que la temperatura superficial varíe rápidamente hacia el interior. Esta tendencia de variación con un gradiente alto dentro de los primeros metros de profundidad es consecuencia del rango de penetración de la temperatura en relación a la profundidad debido a la variación anual de la temperatura del aire (Cai et al., 1988; Liu et al., 2009) y llega a alcanzar entre 15 y 40 metros donde surge un punto de inflexión hacia un gradiente más suave y decreciente; este umbral de transición es conocido como capa activa (Zagorodnov et al., 2006) y es donde se registra la temperatura más baja del perfil en un glaciar templado. A partir de este punto el gradiente térmico adquiere un comportamiento prácticamente lineal (Robin, 1955) acercándose al punto de fusión por presión en tanto más profundo sea. Una temperatura cercana al punto de fusión es lo que caracteriza a los glaciares templados de mediana y baja latitud (Fountain, 2011), mientras que los glaciares polares y circumpolares poseen una temperatura en la parte media superior que comúnmente suele ser menor a $-10^{\circ} \mathrm{C}$ (Paterson, 1981). Con respecto al primer tipo de glaciares, cuyo espesor se ubica entre unas decenas y poco más de cien metros en promedio, Yoshikawa (2013) señala que el perfil de temperatura en su interior se conserva dentro del rango negativo y la línea de tendencia se prolonga hasta el basamento; en con- secuencia, el lecho se encontrará también congelado por contacto y conducción generando lo que podría llamarse permafrost basal. Este principio de congelamiento por contacto ha sido documentado ampliamente desde la perspectiva química y física (Ladino-Moreno et al., 2013).

Tomando en cuenta que los modelos para la estimación presencial de permafrost de alta montaña están restringidos estrictamente a las áreas periglaciares, y que para el caso particular del Citlaltépetl las investigaciones criosféricas más recientes están orientadas a cuantificar el retroceso en la cobertura de su glaciar con el tiempo (Cortés-Ramos y Delgado-Granados, 2012; 2015) y la pérdida de su volumen por medio del balance de energía en su superficie (OntiverosGonzáles et al., 2015), este trabajo intenta aportar una aproximación de las condiciones térmicas al interior del principal cuerpo de hielo en México, ya que la única referencia existente está basada en una muestra obtenida en 1995 a nivel de superficie y otra a $3 \mathrm{mt}$ de profundidad por Palacios y Marcos (1998). Como consecuencia de la estimación de su temperatura vertical, se pretende determinar la posible presencia de permafrost en su lecho.

Considerando que la temperatura del suelo para estimar la presencia de permafrost es función del balance de radiación en la superficie y de su consecuente flujo energético al interior, sin notar el calor geotérmico, al menos las primeras decenas de metros, en esta investigación son tomados en cuenta los mismos parámetros que gobiernan la tasa de temperatura hacia el interior del glaciar de manera vertical y asumiendo un estado constante; es decir, considerando valores promedios de temperatura a través del tiempo así como la consideración de un cuerpo estático, pero al mismo tiempo, son consideradas las propiedades físicas del hielo como la densidad y su difusividad; son contempladas también las oscilaciones de temperatura ocasionadas por la variación temporal.

\section{CARACTERÍSTICAS GENERALES DE LA ZONA DE ESTUdiO}

El volcán Citlaltépetl (Cerro de la estrella en Náhuatl) o Pico de Orizaba es un estratovolcán en fase de reposo que pertenece al Eje Neovolcánico mexicano en su parte más oriental (figura 1a) y se localiza a $200 \mathrm{~km}$ de la Ciudad de México. Posee una altitud de acuerdo con la fuente oficial mexicana sobre estudios territoriales del país (INEGI, 2017) de 5,610 msnm. Es el cono volcánico más alto de América del norte y la tercera cima después del Monte Denali, antes Mckinley (6,190 msnm) y el Monte Logan (5,959 
msnm). Su edad está datada en $\sim 650,000$ años cuando dio inicio la primera etapa de formación a la cual le sucedieron dos fases de evolución más incluyendo la del cráter actual (Macías, 2005). Su cima representa una frontera estatal que divide Puebla al poniente y Veracruz al oriente.

La primera fase de evolución, conocida como Torrecillas, dio inicio hace 650000 años; la segunda, llamada Espolón de Oro, se desarrolló al norte de los remanentes de Torrecillas hace 210000 años. Finalmente, el cráter actual se formó a partir de los restos del Espolón de Oro 16500 años atrás (Macías, 2005). A lo largo de sus distintas fases eruptivas ha cubierto sus laderas con depósitos de piroclastos y flujos de lava; estos flujos son en su mayoría dacita y andesita (Carrasco-Núñez, 2000). La vertiente norte, donde se localiza su glaciar, cuenta con algunos de los más importantes en cuanto a tamaño y cronología: el flujo de dacita y andesita de Jamapa y el de andesita del Paso del Buey. El primero, con un espesor de $400 \mathrm{~m}$ y una edad calculada en $\sim 400000$ años, es producto de la primera fase de formación (Macías, 2005). El segundo flujo pertenece a la segunda etapa de evolución (Macías, 2005); posee $80 \mathrm{~m}$ de grosor y una edad estimada entre 210000 y 40000 años (Carrasco-Núñez, 2000); a esta etapa de desarrollo pertenece también el flujo de dacita y andesita Alpinahua (Macías, 2007). La parte superior del volcán se encuentra cubierta por estas coladas de lava; por encima de éstas existen depósitos sobrepuestos de ceniza y pómez. De acuerdo con Macías (2005), por debajo de la parte media del cono principal estos depósitos están intercalados con distintos flujos y oleadas piroclásticas; Carrasco-Núñez y Rose (1995) les asignaron una antigüedad de 9000 años. Entre los remanentes del Espolón de Oro y los flujos de Alpinahua se forma un valle profundo que comienza por debajo de la parte occidental del glaciar, a una altitud aproximada de $5300 \mathrm{msnm}$, y se prolonga en dirección sur-norte hasta la cota $4600 \mathrm{msnm}$ donde comienza la zona conocida como "la canaleta" y "los laberintos", una zona accidentada en forma de " $v$ " con alto dinamismo por procesos de ladera.

De acuerdo con Heine (1988), en el Citlaltépetl existen vestigios de los avances del Último Máximo Glaciar y de la Pequeña Edad de Hielo, al igual que en las montañas mexicanas de más de 3000 msnm. Estas huellas, indicadas por la presencia de morrenas terminales, comenzaron a formarse hace unos 36000 años y se encuentran principalmente en la vertiente norte del volcán. Con base en la estratigrafía cronológica de Vázquez-Selem (2011), son cinco las fases de morrena terminal (M) que están presentes: M-I a 3200 msnm; M-II a 3900 msnm; M-III a 4000 msnm; M-IV a $4050 \mathrm{msnm}$ y M-V a $4400 \mathrm{msnm}$. Todos estos depósitos morrénicos, muchos de ellos cubiertos en la actualidad por vegetación, por sedimentos de piroclastos y ceniza, o por flujos de lava relativamente recientes, pertenecen a los avances glaciares del final del Cuaternario.

La cubierta vegetal en el entorno del Citlaltépetl está compuesta de bosque mesófilo de montaña por encima de los 600 msnm; por arriba de los 1800 msnm comienza la vegetación de tipo pino-encino con presencia de pino patula (Pinus patula) y ocote (Pinus montezumae) que se extiende hasta cerca de los 3000 msnm donde comienza la población de coníferas que culmina a poco más de 4000 msnm; está compuesta principalmente de pino hartwegii (Pinus hartewii) y oyamel (Abies religiosa). A partir de esta cota están presentes los matorrales y zacatonales que cubren de manera regular hasta cerca de los 4200 msnm; este ecosistema está compuesto principalmente por Calamagrostis tolucensis, Fetusca tolucensis y Muehlenbergia quadridenta (Lauer y Klaus, 1975). Más arriba de este punto sólo se encuentran de manera aislada hasta los 4700 msnm donde comienza el piso supra forestal de carácter crio-orotropical.

En la cara norte y por encima de los 5040 msnm inicia el glaciar que se extiende de forma continua hasta el cráter en la cima del cono. Con una longitud de poco más de mil metros y setecientos cincuenta de ancho en promedio posee una forma que asemeja una elipse con el eje mayor expuesto de sur a norte (figura 1b); su espesor varía entre un par de metros en los límites inferiores hasta 100 metros en su parte occidental, y posee una profundidad media de 23 metros (Ontiveros-González, 2018). Las áreas de mayor profundidad del glaciar, en su porción occidental baja, son el resultado de la acumulación del hielo por encima del valle formado por los remanentes del Espolón de Oro y los flujos de Alpinahua. La figura 2(a) señala la isohipsa 5130 msnm, donde a partir de la exploración con radar GPR se identificó la parte más profunda del glaciar; la figura 2(b) muestra el perfil de sondeo de radar elaborado por encima de la isohipsa 5130. La exploración del perfil comienza en el punto A con coordenadas $19^{\circ} 2^{\prime} 21^{\prime \prime} \mathrm{N}, 97^{\circ} 16^{\prime} 23^{\prime \prime} \mathrm{O}$ y culmina en el punto $B$ con coordenadas $19^{\circ} 2^{\prime} 19.6^{\prime \prime} \mathrm{N}, 97^{\circ} 16^{\prime} 15.5^{\prime \prime} \mathrm{O}$. Puede apreciarse la profundidad máxima alcanzada de 100 metros entre los puntos A y $B$. 
FIGURA 1

ZONA DE ESTUDIO
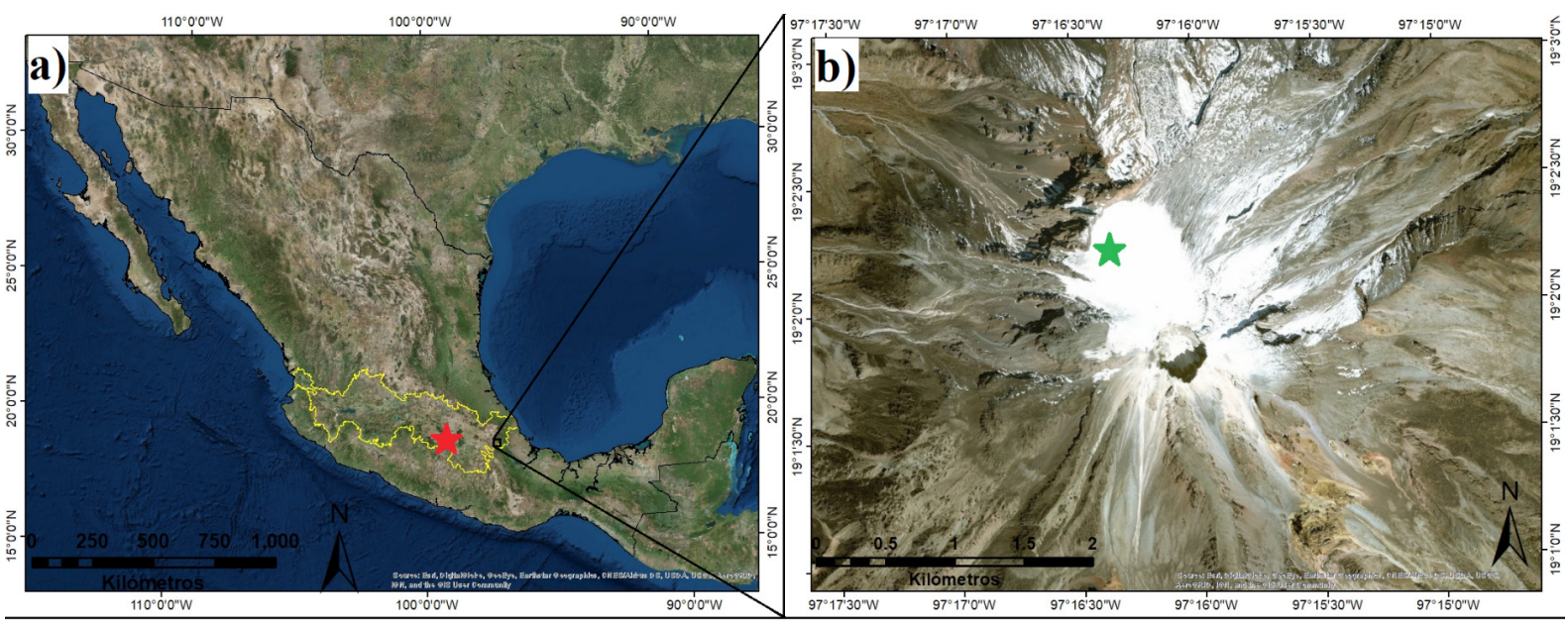

Eje Neovolcánico Mexicano

Zona de estudio

人 Ciudad de México 市 Estación meteorológica "Glaciar"

Fuente: elaboración propia.

FIGURA 2

PROFUNDIDAD MÁXIMA DEL GLACIAR A PARTIR DE EXPLORACIÓN CON RADAR GPR

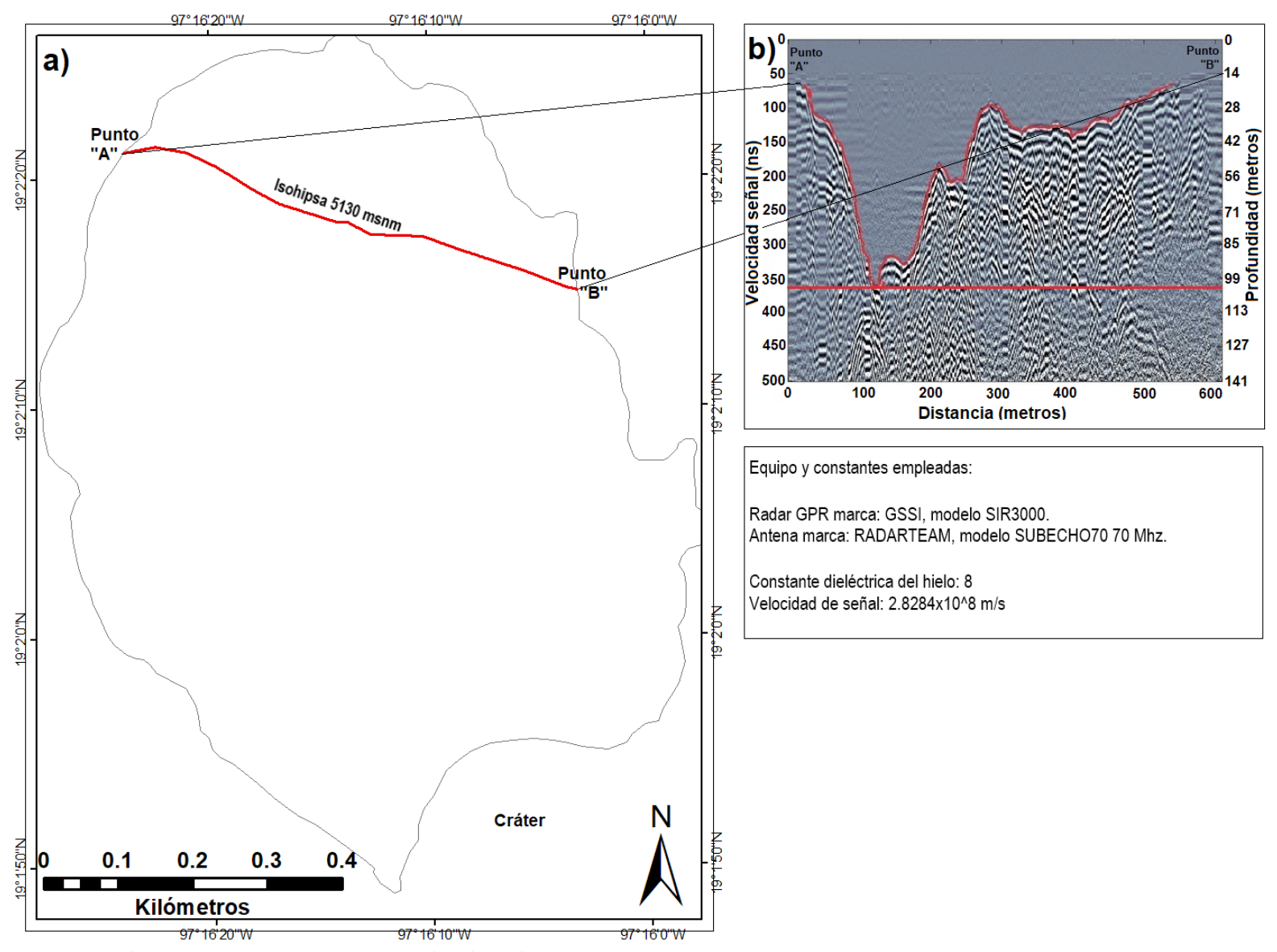

Fuente: modificado a partir de Ontiveros-González (2018). 
El glaciar ha experimentado un franco retroceso desde el final de los años 1950s cuando fue dimensionado y cartografiado por primera vez por Lorenzo (1964). Recientemente, Cortés-Ramos y Delgado-Granados (2015) ubicaron el límite inferior del glaciar a 4980 msnm, contra 4640 msnm que alcanzaba durante el año de 1958. Esta dinámica de retroceso durante las últimas décadas ha dejado porciones de hielo abandonado en la parte baja del valle de "la canaleta" y "los laberintos". Los procesos de ladera del sitio han sepultado con crioclastos y sedimentos de menor diámetro estos remanentes de hielo. Cerca del límite actual del glaciar se aprecian pequeños depósitos morrénicos laterales que han quedado al descubierto por el retroceso actual; estas morrenas están compuestas de pumita y ceniza con contenido de hielo intersticial.

\section{FUENTES Y METODOLOGÍA}

Este trabajo se desarrolla con datos meteorológicos de cuatro años continuos a partir de septiembre del 2006 hasta agosto del 2010 proporcionados por la estación meteorológica automática "Glaciar" ubicada a 5131 msnm e instalada por encima del cuerpo de hielo (figura 1b). Tras poco más de cuatro años de servicio ininterrumpido y debido a cuestiones meteorológicas adversas la estación fue dañada y dejó de funcionar a finales del 2010. La estación está equipada con sensores de temperatura del aire y hielo a 0.5 y 1 metro de profundidad $\left( \pm 0.5^{\circ} \mathrm{C}\right)$, humedad relativa $( \pm 5 \%)$, velocidad y dirección del viento, barómetro digital $( \pm 0.2 \mathrm{hPa})$ y sensor de radiación neta $( \pm 3 \%)$; representando los valores entre paréntesis la precisión de cada sensor. El equipo genera datos cada 30 minutos, así como valores medios diarios durante el periodo de mediciones. La serie ha sido homogeneizada llenando los pocos vacíos diarios que existían mediante el promedio de los meses próximos. De sus datos, Ontiveros-González et al., (2015) calcularon el balance de energía en la superficie durante un periodo de tres años consecutivos de septiembre del 2006 al 2009.

A partir de los registros de temperatura a $0.5 \mathrm{mt}$ de profundidad se calculó la temperatura interna del glaciar considerándolo estacionario, sin embargo, se evitó el empleo de la ecuación (2) de Fourier para disminuir en lo posible el umbral de error por arrojar resultados lineales. Por lo que la estimación de la temperatura interna y su distribución vertical son calculados mediante el método sinusoidal de Kusuda y Achenbach (1965) utilizado ampliamente en el estudio de la temperatura del suelo (p. ej. Givoni y Katz, 1985; Derradji y Aiche, 2014; Yusof et al., 2014;
Upadhyay et al., 2014; Muhammad et al., 2016; Hu et al., 2016; Andújar-Márquez et al., 2016) y que ha sido adecuado para este propósito. El modelo se basa en un principio exponencial en relación a la profundidad y de coseno para la variación de la temperatura la cual tiende a presentar el desfase característico conforme a la profundidad. La validación del modelo ha sido demostrada con gran precisión (Kusuda, 1968), lo que le otorga un alto grado de confianza en este trabajo.

En el modelo son consideradas la conductividad y capacidad calóricas del hielo, así como su difusividad térmica. Paralelamente son empleadas las temperaturas máximas, mínimas, medias de la superficie y su oscilación térmica. De esta manera es posible estimar la temperatura a cualquier profundidad durante el ciclo de un año mediante la expresión:

$T_{(Z, t)}=T_{m}-O A_{s} * e^{-Z \sqrt{\frac{\pi}{365 * \alpha}}} \cos \left[\frac{2 \pi}{365}\left(t-t_{0}-\frac{Z}{2} \sqrt{\frac{365}{\pi * \alpha}}\right)\right]$

Donde:

$T_{(z, t)}$ Representa la temperatura en una profundidad determinada para un tiempo dada en ${ }^{\circ} \mathrm{C}$.

$T_{m}$ Es la temperatura media anual de la superficie en ${ }^{\circ} \mathrm{C}$.

$O A_{s}$ Es la oscilación térmica anual de la superficie en ${ }^{\circ} \mathrm{C}$.

$Z$ Es la profundidad de interés en metros.

$t$ Es el tiempo en días en el cual se desea conocer la temperatura.

$t_{0}$ Es el desfase en días, número del día en el cual la temperatura superficial es mínima.

Constantes empleadas en la ecuación:

$\pi=3.14$

$\alpha=1.09$ (difusividad térmica del hielo a $0^{\circ} \mathrm{C}$ ).

Del universo de datos de cuatro años continuos se separaron los correspondientes al verano e invierno y se obtuvieron sus promedios. Posteriormente se promediaron los valores de la parte central del invierno y del verano de cada año para obtener el valor más representativo para la estación más fría y más cálida; de igual manera se obtuvo la temperatura media anual. La temperatura fue calculada en rangos de 5 metros de profundidad llegando hasta 100 que se encontraron como máximo espesor del glaciar. Los valores en profundidad fueron tabulados y graficados para señalar el comportamiento térmico promediado entre 
los cuatro años durante el verano y el invierno. Posteriormente se elaboró una capa ráster del área del glaciar y se interpolaron los valores de temperatura calculados con base en las profundidades estimadas; el proceso se desarrolló por medio de la herramienta Map Algebra de $\operatorname{ArcGis}^{\circledR} 10.4$ para obtener así el mapa de temperatura basal. Finalmente se consideró que la temperatura en la base del glaciar se asignaría al lecho del substrato volcánico para determinar la posible presencia de permafrost.

\section{RESULTADOS}

Una vez aplicada la ecuación 3 de Kusuda y Achenbach (1965) para cada profundidad en rangos de 5 metros, los resultados de temperatura para la estación más fría y más cálida del año, así como la media anual se muestran en la tabla 1 . En la tabla aparecen sombreadas las celdas correspondientes a la profundidad media del glaciar (23 mt), así como los puntos de profundidad máxima (100 mt).

TABLA 1

TEMPERATURA ESTIMADA DEL GLACAIR EN RELACIÓN A LA PROFUNDIDAD

\begin{tabular}{|c|c|c|c|}
\hline \multirow{2}{*}{$\begin{array}{c}\text { Profundidad } \\
\text { (mt) }\end{array}$} & \multicolumn{3}{|c|}{ Temperatura $\left({ }^{\circ} \mathrm{C}\right)$} \\
\cline { 2 - 4 } & Invierno & Verano & Media anual \\
\hline-5 & -4.39 & -0.15 & -2.27 \\
\hline-10 & -4.00 & -0.99 & -2.50 \\
\hline-15 & -3.46 & -1.53 & -2.50 \\
\hline-20 & -3.12 & -1.88 & -2.50 \\
\hline-25 & -2.89 & -2.10 & -2.50 \\
\hline-30 & -2.75 & -2.25 & -2.50 \\
\hline-35 & -2.66 & -2.34 & -2.50 \\
\hline-40 & -2.60 & -2.40 & -2.50 \\
\hline-45 & -2.56 & -2.43 & -2.50 \\
\hline-50 & -2.54 & -2.46 & -2.50 \\
\hline-55 & -2.52 & -2.47 & -2.50 \\
\hline-60 & -2.51 & -2.48 & -2.50 \\
\hline-65 & -2.50 & -2.49 & -2.50 \\
\hline-70 & -2.49 & -2.49 & -2.49 \\
\hline-75 & -2.49 & -2.49 & -2.49 \\
\hline-80 & -2.49 & -2.49 & -2.49 \\
\hline-85 & -2.48 & -2.49 & -2.49 \\
\hline-90 & -2.48 & -2.49 & -2.49 \\
\hline-95 & -2.48 & -2.49 & -2.49 \\
\hline-100 & -2.48 & -2.49 & -2.49 \\
\hline
\end{tabular}

Fuente: Elaboración propia.
Del mismo modo, la gráfica de la figura 3 muestra la distribución de cada temperatura calculada.

Los resultados obtenidos muestran una clara disminución del gradiente conforme aumenta la profundidad dentro de los primeros 40 metros. Hasta este punto se aprecia una clara incidencia de los efectos radiativos sobre la superficie y la influencia de la temperatura del aire condicionando que el gradiente térmico alcance los valores más significativos en las temperaturas del verano y el invierno (figura 2). Esta marcada variación ha sido enfatizada por Palacios y Marcos (1998) quienes reportaron que durante una medición llevada a cabo durante el mes de octubre de 1995 la temperatura superficial partía de $0^{\circ} \mathrm{C}$ hasta alcanzar $-1.3^{\circ} \mathrm{C}$ a tan sólo 3 metros de profundidad. El límite de penetración de la temperatura cercano a los 40 metros de profundidad marca el punto de transición correspondiente a la capa activa señalada previamente para los glaciares templados. A partir de este nivel la variación tiende a ser mucho menor hasta cerca de los $60 \mathrm{mt}$ de profundidad donde converge el periodo más frío y más cálido del año, punto donde se considera ya inexistente la oscilación térmica. Entre la superficie y 15 metros de profundidad es la zona que presenta la máxima variación térmica cercana a $0.5^{\circ} \mathrm{C}$ en promedio cada 5 metros tanto para el verano como el invierno (Tabla 1).

Por su parte, la temperatura media anual desciende claramente desde la superficie hasta $10 \mathrm{mt}$ de profundidad. La temperatura media es casi constante a parir de $10 \mathrm{mt}$ y hasta la base del glaciar, partiendo de $-2.50^{\circ} \mathrm{C}$ con un gradiente casi nulo hasta los $100 \mathrm{mt}$ donde alcanza una temperatura de $-2.49^{\circ} \mathrm{C}$. Esto ocasiona que se presente una línea de tendencia casi vertical. Este comportamiento obedece a las características relativamente someras del glaciar, por lo que el efecto de fusión por presión debería advertirse de manera más clara en un glaciar de mayor profundidad, tal como lo señalan Paterson (1981) y Yoshikawa (2013).

La figura 4 muestra el mapa de distribución de la temperatura basal del glaciar con sus valores extremos respectivos para cada periodo del año, y representa al mismo tiempo el modelamiento térmico del lecho sobre el que descansa. El modelo se aprecia tanto en vista azimutal como en perspectiva tridimensional. El color más oscuro señala una profundidad más superficial y a la vez la temperatura más fría, mientras que el color más claro indica una mayor profundidad y una temperatura menos fría. 
FIGURA 3

DISTRIBUCIÓN VERTICAL DE TEMPERATURAS EN EL GLACIAR

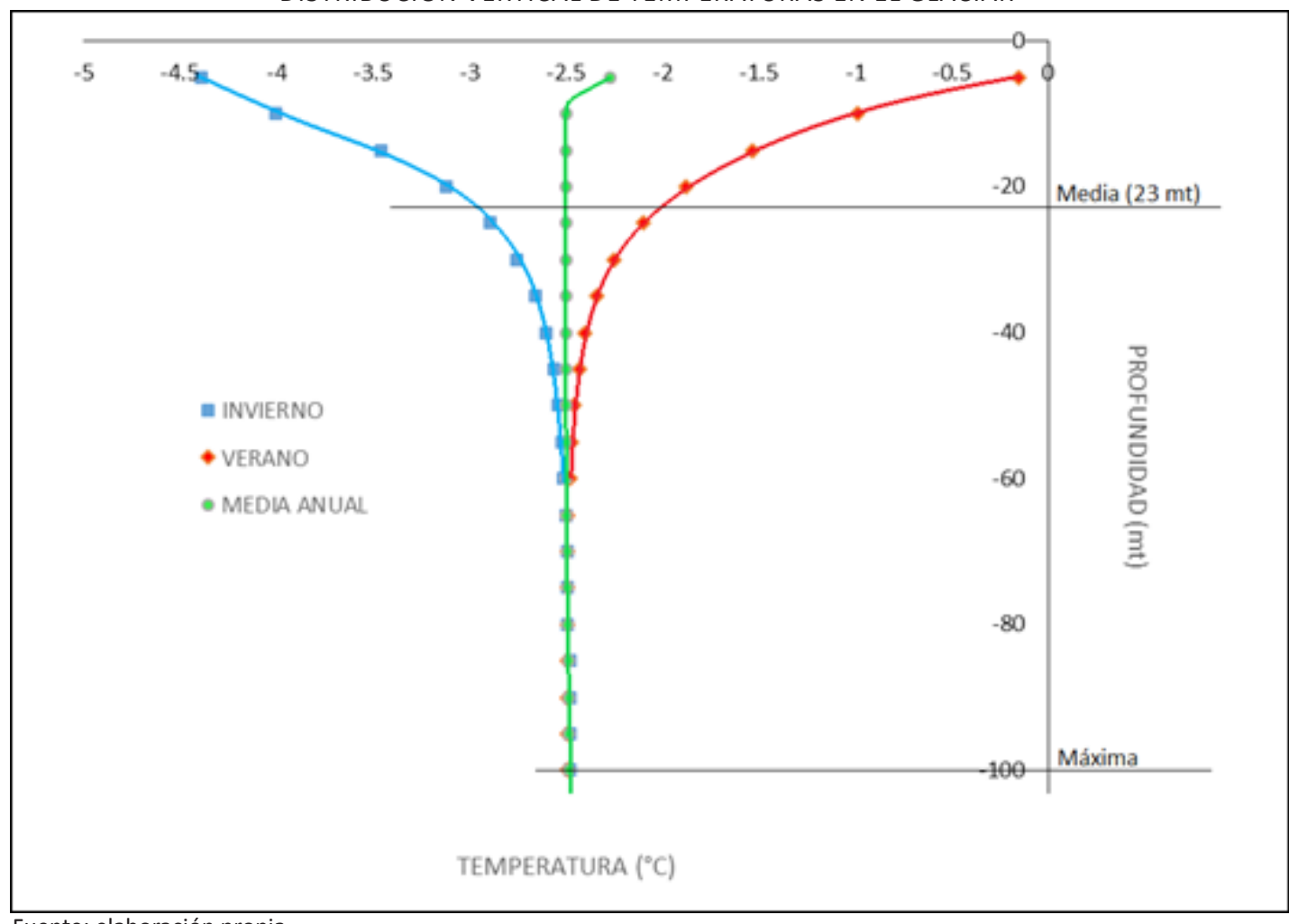

FIGURA 4

MAPA DE DISTRIBUCIÓN DE TEMPERATURAS EN LA BASE DEL GLACIAR
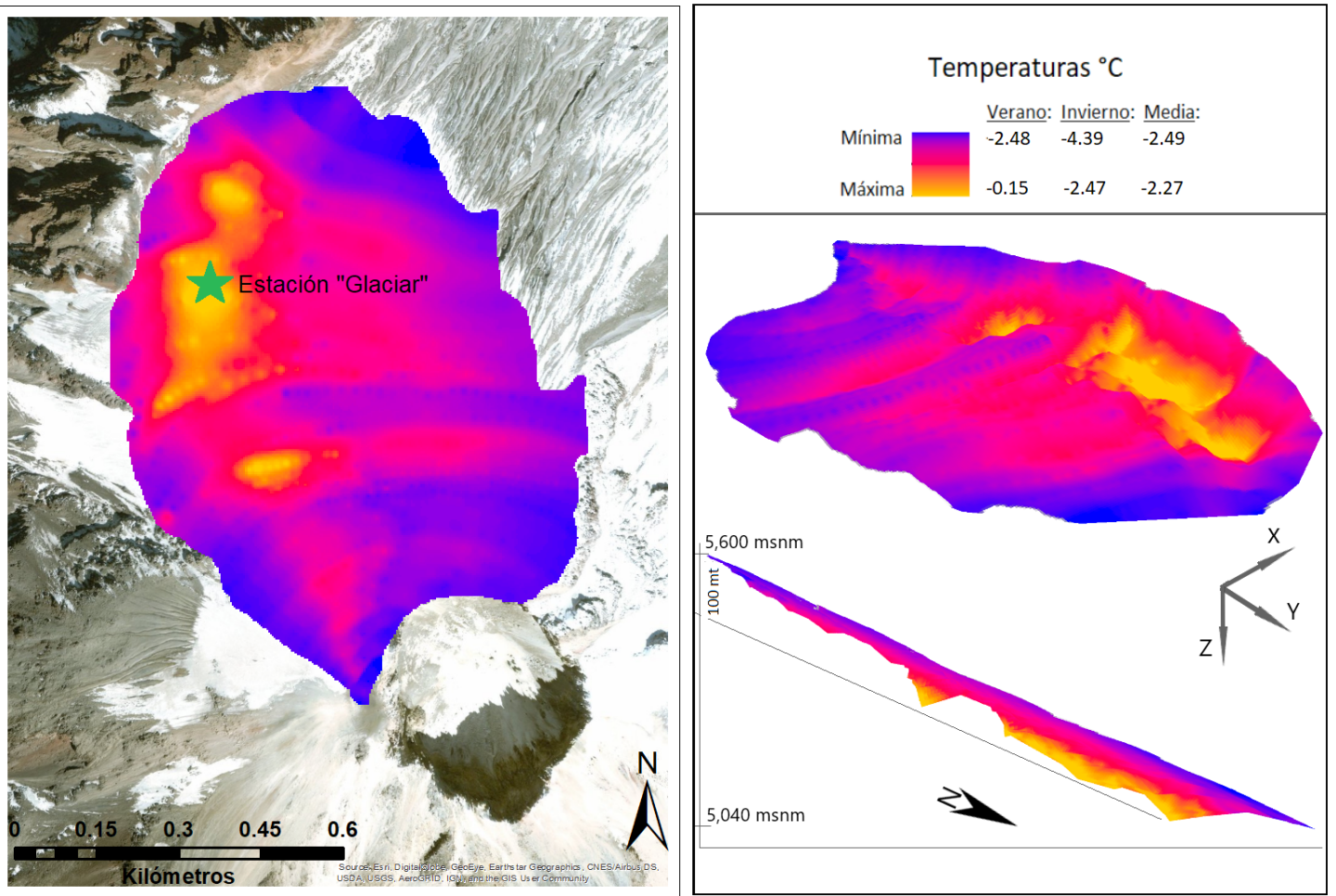

Fuente: elaboración propia. 


\section{Discusıón}

Determinar la presencia de permafrost en la base de los glaciares no es una tarea fácil, sobre todo por el espesor de los cuerpos de hielo polar y circumpolar; por esta razón existen discrepancias con respecto a la presencia o no de permafrost subglaciar. Por ejemplo, French (2007) señala que, por debajo de la cubierta glaciar, toda la Antártica está bajo condiciones de permafrost, mientras que Bockheim y Hall (2002) indican que su presencia está limitada a pequeñas áreas debajo de la cubierta de hielo, al igual que para el caso de Groenlandia. Esto último podría obedecer en parte, como se ha señalado, a que los glaciares de grandes dimensiones y profundidad, son a menudo politermales, como lo menciona Kotlyakov (1984), y es común la presencia de agua en su base por los efectos de presión y fricción. A este respecto, Dyke (1993) encontró una temperatura de $3^{\circ} \mathrm{C}$ por debajo de $1100 \mathrm{~m}$ de hielo glaciar. Sin embargo, tal como lo señala Dobiński (2012), las condiciones de temperatura en glaciares no profundos, propician la presencia de permafrost en su base. Esto es enfatizado por los señalamientos de Gow et al., (1979), Koerner y Fisher (1979) y Gemmell et al., (1986), quienes confirman que los pequeños glaciares de latitudes altas y los de alta montaña poseen un basamento congelado por los efectos de temperaturas negativas en su parte inferior; todos estos con un espesor medio de decenas de metros.

Las condiciones orográficas, litológicas y sedimentarias de la parte alta del Citlaltépetl han propiciado que la formación del "Glaciar Norte" se haya desarrollado por encima de un lecho muy irregular y accidentado, lo que repercute en su espesor a lo largo de distintos puntos. Mientras que el valor promedio para todo el cuerpo de hielo oscila en 23 metros, el valle profundo ubicado en su costado occidental, el cual se prolonga hasta cotas inferiores, propicia que alcance los cien metros de profundidad. Este valor atípico en la profundad del glaciar, particularmente para aquellos de latitudes medias y tropicales, no es producto de un alto índice de precipitación nival en el sitio, sino que debió ser causa de la reptación y la acumulación por gravedad del hielo a lo largo de la formación y evolución de este glaciar.

Los valores de temperatura encontrados en los glaciares circumpolares y polares son inferiores a $-10^{\circ} \mathrm{C}$, al menos en su parte media superior, tal como lo señala Paterson (1981), y difieren considerablemente de las temperaturas que se presentan en los glaciares de alta montaña de latitudes medias y bajas. El caso del glaciar del Citlaltépetl, en la base de su espesor medio de $23 \mathrm{~m}$, refleja la incidencia de la temperatura superficial durante el año (figura 3); este rango estacional de temperaturas va de $-2.9^{\circ} \mathrm{C}$ durante el invierno a $-2^{\circ} \mathrm{C}$ en el verano. Si bien la amplitud térmica anual a $23 \mathrm{~m}$ de profundidad es sólo de $0.9^{\circ} \mathrm{C}$, este valor refleja el grado de penetración de la temperatura estacional, y al mismo tiempo señala sus características térmicas casi homogéneas.

La estimación de la temperatura del "Glaciar Norte" cercana a $-2.5^{\circ} \mathrm{C}$ tanto para su espesor medio, como para los puntos de mayor profundidad, y la consecuente presencia de permafrost subglaciar con el mismo valor térmico, coinciden de cerca con los valores señalados por French y Shur (2010) y Barry (2017) para regiones de mediana latitud. Esta estimación representa una aproximación a las condiciones actuales del ambiente periglaciar en regiones tropicales por arriba de 5000 msnm, particularmente en cuanto a la determinación de permafrost subglaciar. Por lo tanto, estos resultados pueden ser un referente para trabajos criosféricos de latitudes medias e intertropicales.

Paralelamente a lo anterior, los valores de temperatura subsuperficial y al interior del "Glaciar Norte" que se encontraron en este trabajo confirman que la retracción actual de este glaciar no es una consecuencia estricta de la temperatura del aire en su entorno, sino que obedece más a los procesos asociados con la energía radiativa en su superficie, tal como lo señalan Ontiveros-González et al., (2015). Este forzamiento radiativo y su consecuente efecto de sublimación queda de manifiesto con la presencia común de penitentes de hasta un metro de altura durante los periodos de primavera y verano.

\section{CONCLUSIONES}

El poco espesor de la masa de hielo condiciona que el perfil de temperatura interna del glaciar del Citlaltépetl se mantenga dentro del rango de congelamiento sin mostrar un proceso de fusión por presión. Las condiciones de temperatura promediadas a lo largo de cuatro años permiten sugerir la posible existencia de permafrost basal por debajo del glaciar debido a que, además del efecto aislante del hielo ante la radiación solar incidente, el basamento en su parte media (23 metros) y más profunda (100 metros) se conserva cerca de $-2.5^{\circ} \mathrm{C}$ en promedio a lo largo de los cuatro años, sin registrar la mínima oscilación de temperatura. Esta temperatura es transferida por conducción al contacto con el lecho sobre el que descansa. Incluso las partes menos profundas 
que son mayormente influenciadas por los rangos de temperatura estacional no alcanzan a cruzar el límite del punto de fusión a lo largo del año. Dado que se trataría de un permafrost en el límite del régimen térmico (por debajo de $0^{\circ} \mathrm{C}$ según French $(2007$, p. 83); o muy próximo a $-2{ }^{\circ} \mathrm{C}$ de French y Shur (2010) y Barry (2017); o bien variable entre $-1,5$ y -5 으 en los denominados permafrost discontinuos, el per-

\section{Bibliografía}

Ahrens, C. D. (2006). Meteorology Today, An Introduction to Weather, Climate and Environment (8. a ed.). California, USA: Thompson Brooks/Cole Publishing.

Andújar Márquez, J. M., Martínez Bohórquez, M. Á., y Gómez Melgar, S. (2016). Ground thermal diffusivity calculation by direct soil temperature measurement. Application to very low enthalpy geothermal energy systems. Sensors, 16(306), 1-13.

Arya, P. S. (2001). Introduction to micrometeorology (Vol. 79). Academic press.

Barry, R.G. (2017). The Arctic cryosphere in the twentyfirst century, Geographical Review, 107, 1, 69-88.

Bennett, M. M., y Glasser, N. F. (eds.) (2011). Glacial geology: ice sheets and landforms (2. a ed.). West Sussex, UK: John Wiley \& Sons.

Blatter, H., y Haeberli, W. (1984). Modelling temperature distribution in Alpine glaciers. Annals of Glaciology, 5(1), 18-22.

Bockheim J.G., y Hall K.J., (2002). Permafrost, activelayer dynamics and periglacial environments of continental Antarctica. S. Afr. J. Sci., 98, 82-90.

Cai, B., Huang M., y Xie Z. (1988). A preliminary research on the temperature in deep boreholes of Glacier No. I, Urumqi headwaters. Kexue Tongbao, 33(24), 2054-2056.

Carrasco-Núñez, G. (2000). Structure and proximal stratigraphy of Citlaltepetl volcano (Pico de Orizaba), Mexico. En Cenozoic tectonics and volcanism of Mexico (pp. 247-262), vol. 334.

Carrasco-Núñez, G., y Rose, W. I. (1995). Eruption of a major Holocene pyroclastic flow at Citlaltépetl volcano (Pico de Orizaba), México, 8.5-9.0 ka. Journal of volcanology and geothermal research, 69(3), 197-215.

Ceballos-Liévano, J. L., Rodríguez-Murcia, C. E., y RealNúñez, E. L. (eds.) (2012). Glaciares de Colombia, más que montañas con hielo. Bogotá: IDEAM. mafrost basal sería de carácter limitado en función de las características del substrato afectado, siendo más factible en pumicitas y menos en rocas volcánicas masivas. El congelamiento del lecho, al menos en su porción más somera sugiere que el permafrost presente alcance su propia base hasta el punto en el que el calor geotérmico, obligue a que la curva de temperatura cruce hacia el umbral positivo.

Cortés-Ramos, J., y Delgado-Granados, H. (2012). The recent retreat of Mexican glaciers on Citlatepetl volcano detected using ASTER data. The Cryosphere Discussions, 6, 3149-3176.

Cortés-Ramos, J., y Delgado-Granados, H. (2015). Reconstruction of glacier area on Citlaltépetl volcano, 1958 and implications for Mexico's deglaciation rates. Geofísica internacional, 54(2), 111-125.

Cuffey, K. M., y Paterson, W. S. B. (2010). The physics of glaciers (4. a ed.). Oxford, UK: Elsevier, Inc.

Demek, J. (1994). Global warming and permafrost in Eurasia: a catastrophic scenario, Geomorphology, 10, 317-329.

Derradji, M., y Aiche, M. (2014). Modeling the soil surface temperature for natural cooling of buildings in hot climates. Procedia Computer Science, 32, 615-621.

Dobiński, W. (2012). The cryosphere and glacial permafrost as its integral component. Central European Journal of Geosciences, 4(4), 623-640.

Dyke, A.S., (1993). Landscapes of cold-centred Late Wisconsin ice caps, Arctic Canada. Prog. Phys. Geog. 17, 223-247.

Fountain, A. (2011). Temperate glaciers. In: V. P. Singh, U. K. Haritashya, P. Singh (eds.). Encyclopedia of snow, ice and glaciers (pp. 11455). Berlin: Springer.

French, H.M. (1976). The periglacial environment (1. a ed.). London and New York: Longman.

French, H.M. (2007). The periglacial environment (3. ed.). Chichester, New Jersey: Wiley and Sons.

French, H.; Shur, Y. (2010). The principles of criostratigrapy, Earth-Science Reviews, 101, 190-206.

Gemmell, A.M.D., Sharp, M.J., Sugden, D.E., (1986). Debris from the basal ice of the Agassiz ice-cap, Ellesmere Island, Arctic Canada. Earth Surf. Process Landf. 11, 123-130. 
Givoni, B., y Katz, L. (1985). Earth temperatures and underground buildings. Energy and Buildings, 8(1), 15-25.

Gow, A.J., Epstein, S., Sheehy, W., (1979). On the origin of stratified debris in ice cores from the bottom of the Antarctic ice sheet. J. Glaciol., 23, 185-192.

Grenfell, T. C. (2011). Albedo. En Encyclopedia of Snow, Ice and Glaciers (pp. 23-35). Berlin: Springer.

Heine, K. (1988). Late Quaternary glacial chronology of the Mexican volcanoes. Die Geowissenschaften, 7, 197-205.

Hu, G., Zhao, L., Wu, X., Li, R., Wu, T., Xie, C., y Cheng, G. (2016). An analytical model for estimating soil temperature profiles on the Qinghai-Tibet Plateau of China. Journal of Arid Land, 8(2), 232-240.

Instituto Nacional de Estadística, Geografía e Informática, INEGI (2017). Anuario estadístico y geográfico de los Estados Unidos Mexicanos. Aguascalientes, Ags: INEGI.

Koerner, R.M., Fisher, D.A. (1979). Discontinuous flow, ice texture and dirt content in the basal layers of Devon Island ice cap. J. Glaciol. 23, 209-222.

Kotlyakov, V.M. (1984). Glaciological dictionary. Leningrad: Gidrometeoizdat. 528 pp.

Kusuda, T. (1968). Least squares analysis of annual Earth temperatures cycles for selected stations in the United States. Gaithersburg, USA: National Bureau of Standards.

Kusuda, T. y Achenbach, P. R. (1965). Earth temperature and thermal diffusivity at selected stations in the United States. Building Research Division: National Bureau of Standards, Washington, DC.

Ladino Moreno, L. A., Stetzer, O., y Lohmann, U. (2013). Contact freezing, a review of experimental studies. Atmospheric Chemistry and Physics, 13(19), 9745-9769.

Lauer, W., y Klaus, D. (1975). Geoecological investigations on the timberline of Pico de Orizaba, Mexico. Arctic and Alpine Research, 7, 315-330.

Liu, Y., Hou, S., Wang, Y., y Song, L. (2009). Distribution of borehole temperature at four high-altitude alpine glaciers in central Asia. Journal of Mountain Science, 6(3), 221-227.

Lorenzo, J. L. (1964). Los glaciares de México (2ª ed.). Monografías del Instituto de Geofísica. México: Universidad Nacional Autónoma de México.
Macías, J. L. (2005). Geología e historia eruptiva de algunos de los grandes volcanes activos de México. Boletín de la Sociedad Geológica Mexicana, 57(3), 379-424.

Macías, J. L. (2007). Geology and eruptive history of some active volcanoes of México, Geological Society of America Special Papers, 422, 183-232.

Muhammad, I. S., Baharun, A., y Ibrahim, H. S. (2016). Investigation of Ground Temperature for Heat Sink Application in Kuching, Sarawak, Malaysia. Journal of Civil Engineering, 7(1), 20-29.

Ontiveros-González, G. (2018). Estudios de la dinámica glacial del Glaciar Norte del volcán Citlaltépetl. Tesis de doctorado. Posgrado en Ciencias de la Tierra. Cd. De México: UNAM.

Ontiveros-González, G., Delgado-Granados, H., y Cortés-Ramos, J. (2015). Surface Energy Balance model for high-altitude glacial system at $19^{\circ} \mathrm{N}$ on Glaciar Norte, Mexico. Geofísica internacional, 54(4), 299-314.

Palacios, D., y Marcos, J. D. (1998). Glacial retreat and its geomorphologic effects on Mexico's active volcanoes, 1994-95. Journal of Glaciology, 44(146), 63-67.

Paterson, W.S.B. (1981). The physics of glaciers. (2. ed.). New York: Pergamon Press.

Ramírez, A. Z., y Muñoz, C. B. (2012). Albedo effect and Energy efficiency of cities. In Sustainable Development-Energy, Engineering and TechnologiesManufacturing and Environment. Shangai, China: InTech, 3-18.

Rivas, R. y Caselles V. (2004). A simplified equation to estimate spatial reference evaporation from remote sensing based surface temperature and local meteorological data. Remote Sensing of Environment, 93, 68-76.

Rivas, R., y Ocampo, D. (2009). Comportamiento del balance de energía en un cultivo de Avena sativa L. En Estudios de la zona no saturada del suelo (pp. 336-343).Barcelona: Área Temática III.

Robin, G. (1955). Ice Movement and Temperature Distribution in Glaciers and Ice Sheets. Journal of Glaciology, 2 18, 523-532.

Upadhyay, G., Kämpf, J. H., y Scartezzini, J. L. (2014). Ground temperature modelling: The case study of Rue des Maraîchers in Geneva. In Proceedings of the EuroGraphics 2014 on Urban Data Modelling 
and Visualisation (pp. 1-6). EuroGraphics Digital Library.

Van Den Broeke, M., Fettweis, X., y Mölg T. (2011). Surface energy balance. In Encyclopedia of Snow, Ice and Glaciers (pp. 23-35). Berlin: Springer.

Vázquez-Selem, L., (2011). Las glaciaciones en las montañas del centro de México. En M. Caballero y B. Ortega Guerrero (Eds.), Escenarios de cambio climático: registros del Cuaternario de América Latina I (pp.215-238). México: Universidad Nacional Autónoma de México.
Yoshikawa, K. (ed.) (2013). Permafrost in our time: community-based permafrost temperature archive. Fairbanks AK: University of Alaska Fairbanks Permafrost Outreach Program.

Yusof, T. M., Anuar, S., e Ibrahim, H. (2014). Numerical investigation of ground cooling potential for Malaysian climate. International Journal of Automotive and Mechanical Engineering, 10, 2081-2090.

Zagorodnov, V., Nagornov, O., y Thompson, L. G. (2006). Influence of air temperature on a glacier's active-layer temperature. Annals of Glaciology, 43(1), 285-291. 